\title{
Nuclear data adjustment based on the interpretation of post-irradiation experiments with the DARWIN2.3 package
} \author{
Romain Eschbach ${ }^{1}$ \\ ${ }^{1}$ CEA, DEN, DER, SPRC, Cadarache, 13108 Saint-Paul-Lez-Durance, France \\ 2 CEA, DEN, DER, SESI, Cadarache, 13108 Saint-Paul-Lez-Durance, France \\ 3 AREVA NC, BU Recyclage, Paris, France
}

Axel Rizzo ${ }^{1, *}$, Claire Vaglio-Gaudard ${ }^{2}$, Gilles Noguere ${ }^{1}$, Julie-Fiona Martin ${ }^{3}$, Vanessa Vallet ${ }^{1}$, and

Received: 30 October 2017 / Received in final form: 20 February 2018 / Accepted: 17 May 2018

\begin{abstract}
DARWIN2.3 is the French reference package dedicated to fuel cycle applications, computing fuel inventory as well as decay heat, neutron emissions, $\alpha, \beta$ and $\gamma$ spectra. The DARWIN2.3 package fuel inventory calculation was experimentally validated with Post-Irradiation Experiments (PIEs), mainly consisting in irradiated fuel pellets analysis. This paper presents a method to assimilate these integral trends for improving nuclear data. In this study, the method is applied to ${ }^{137} \mathrm{Cs} /{ }^{238} \mathrm{U}$ concentration ratio. Results suggest an increase of the JEFF-3.1.1 ${ }^{235} \mathrm{U}$ cumulated thermal fission yield in ${ }^{137} \mathrm{Cs}$ by $(+3.8 \pm 2.1) \%$, from $6.221 \mathrm{E}-02$ to $6.460 \mathrm{E}-02 \pm 2.1 \%$.
\end{abstract}

\section{Introduction}

${ }^{137} \mathrm{Cs}$ is a nuclide of interest for the nuclear fuel cycle [1] mostly because it is a convenient burnup indicator thanks to its $\gamma$-ray emission. It is therefore of major importance to compute its concentration in nuclear fuel as a function of the combustion rate as accurately as achievable.

DARWIN2.3 [2] is the French reference package for fuel cycle applications. It solves the Boltzmann and Bateman equations to compute fuel cycle parameters, at any irradiation and cooling time. A package is defined by a nuclear data library, one or several computer codes, and one or several calculation schemes. For DARWIN2.3, nuclear data used come from the JEFF-3.1.1 evaluation [3].

DARWIN2.3 includes both deterministic transport codes APOLLO2 [4] (for light water reactors) and ERANOS2 [5] (for fast reactors), which provide neutron data to the DARWIN/PEPIN2 depletion solver [6]. APOLLO2 and DARWIN/PEPIN2 codes are developed by CEA/DEN with the support of its industrial partners, AREVA and EDF. These neutron data are self-shielded cross sections libraries and multigroup neutron fluxes as a function of burnup.

In addition, data such as multigroup activation cross sections at infinite dilution, a full filiation chain, and specific nuclear constants are used in the DARWIN/ PEPIN2 solver, directly taken from JEFF-3.1.1. This

\footnotetext{
* e-mail: axel.rizzo@cea.fr
}

allows taking into account isotopes and reactions that are not described in the simplified filiation chains used in APOLLO2 or ERANOS2.

The reference calculation scheme used for DARWIN2.3 PWR calculations, called CYCLE2008-PWR [2], is based on the recommended APOLLO2.8 calculation scheme REL2005 [4] used for neutron transport calculations. These two calculation schemes mainly differ in the flux solver used (Probability Collision method instead of the Method Of Characteristics) and energy collapsing.

The DARWIN2.3 package has been experimentally validated for light water reactors for the material balance and decay heat calculation [2]. It has also been experimentally validated for sodium fast reactors for the material balance of the main actinides and fission products involved in burn up-credit calculations [7].

The experimental validation of the DARWIN2.3 package for material balance calculation was performed for a large range of burnup from 10 to $85 \mathrm{GWd} / \mathrm{t}$ for $\mathrm{UOX}$ fuels and from 10 to $60 \mathrm{GWd} / \mathrm{t}$ for MOX fuels. Table 1 displays Calculation-to-Experimental values for the ${ }^{137} \mathrm{Cs}$ concentration that will be used for this study.

One can observe a slight underestimation of ${ }^{137} \mathrm{Cs}$ for UOX fuels whereas ${ }^{137} \mathrm{Cs}$ is better calculated in MOX fuel. It is worth clarifying that the $\sigma$ uncertainty associated to the Calculation-to-Experimental values gathers:

- the precision on the fuel temperature, taken at $\pm 50^{\circ} \mathrm{C}$ at $1 \sigma$ (systematic contribution),

- the precision on the moderator temperature, taken at $\pm 2{ }^{\circ} \mathrm{C}$ at $1 \sigma$ (systematic contribution), 
Table 1. Results of the DARWIN2.3 experimental validation of ${ }^{137} \mathrm{Cs}$ in PWR fuels (1 $\sigma$ standard deviation) [2].

\begin{tabular}{lccc}
\hline PIE and fuel type & Burnup & \multicolumn{2}{c}{${ }^{137} \mathrm{Cs} /{ }^{238} \mathrm{U}$} \\
\cline { 2 - 4 } & $(\mathrm{GWd} / \mathrm{t})$ & $(C / E)-1[\%]$ & $\sigma[\%]$ \\
\hline & 20 & -3.5 & 2.1 \\
Bugey - Fessenheim & 25 & -5.4 & 2.0 \\
(3.1\%-enriched UOX fuel) & 40 & -7.1 & 2.1 \\
& 50 & -4.7 & 2.0 \\
& 60 & -5.6 & 2.3 \\
& & & \\
Gravelines (4.5\%-enriched & 40 & -7.5 & 2.2 \\
UOX fuel) & 50 & -6.4 & 2.3 \\
& 60 & -6.0 & 2.1 \\
& & & \\
Malibu (4.3\%-enriched & 70 & 1.3 & 2.1 \\
UOX fuel) & & & \\
& & & \\
Dampierre (6.7\% Pu & 40 & -1.5 & 1.4 \\
amount MOX) & 52 & -0.7 & 1.5 \\
& 58 & 1.5 & 1.3 \\
\hline
\end{tabular}

- the local burnup characterization, corresponding to the uncertainty on neodymium fission yields - used as burnup indicators - taken at $\pm 2 \%$ at $1 \sigma$ (systematic contribution),

- the measurement uncertainty on concentration ratio at $1 \sigma$ (statistic contribution).

DARWIN2.3 accuracy can be improved by identifying sources of biases and uncertainties [8]. In this framework, this paper presents an integral data assimilation method to improve nuclear data involved in the buildup of nuclides of interest for the fuel cycle.

Next section will present this method, and Section 3 will illustrate an application to the ${ }^{137} \mathrm{Cs}$ case. Results will be presented and discussed in Section 4.

\section{Integral data assimilation method}

To assimilate the integral trends of the DARWIN2.3 package, a Bayesian approach was considered. The CONRAD $[9,10]$ code was used for this study. The successive steps of the study are presented in the following sections.

\subsection{Experimental correlation matrix, and scheme- related uncertainties}

Since the experiments taken from the database are correlated, the AGS code [11] method was used to compute an experimental correlation matrix, combining statistic and systematic uncertainties.

The sources of uncertainties considered are the ones mentioned in Section 1, except the uncertainty on the local burnup characterization (neodymium fission yields), which was marginalized (see Sect. 2.3).

Moreover, since DARWIN2.3 is a package using deterministic solvers, methodological approximations (self-shielding, spatial discretization...) may introduce numerical biases on the calculated parameters. Thus, in addition to the sources of uncertainties previously mentioned, dedicated studies were carried out to provide an order of magnitude of these numerical biases.

A comparative pin-cell depletion calculation was carried out between APOLLO2 with the CYCLE2008 calculation scheme and the reference stochastic code TRIPOLI4 [12], which can perform depletion calculation thanks to a coupling with the MENDEL depletion solver [13]. The ensuing discrepancies on material balance of fission products and main actinides have been found to be of the order of $1 \%$ or less; they will be considered as $1 \sigma$ systematic uncertainty on fuel inventory calculation with the DARWIN2.3 package for this study.

The effect of the resonant up-scattering phenomenon [14], which can be simulated with APOLLO2, can be considered as a modelling bias on the deterministic calculation scheme as well. A comparative pin-cell depletion study was carried out with and without this modelling option to assess its impact on fuel inventory calculation. The ensuing discrepancies on material balance have been found to be of the order of $1 \%$ or less, and they will also be considered as $1 \sigma$ systematic uncertainty.

To provide an experimental correlation matrix, each systematic contribution was considered as a unit normalization factor with an associated uncertainty corresponding to the systematic contribution, hence:

$$
\left\{\begin{array}{l}
E^{\prime}=E \prod_{i} \lambda_{i}=E \\
\Delta E^{\prime}=\sqrt{(\Delta E)^{2}+\sum_{i}\left(\Delta_{i}\right)^{2}},
\end{array}\right.
$$

where:

- $E=$ experimental value before normalization,

- $E^{\prime}=$ experimental value after normalization,

$-\lambda_{i}=1 \pm \Delta_{i}$ is the unit normalization factor associated with the $i$ th source of uncertainty,

$-\Delta_{i}=$ standard deviation of $\lambda_{i}$.

Eventually, one can build the $M_{E}$ experimental correlation matrix with the AGS code [11] method:

$$
M_{E}=D+S \cdot S^{T},
$$

where:

- $D$ is a diagonal matrix filled with experimental variances (statistical uncertainty),

- $S$ is a rectangular matrix - number of experiment $\times$ number of systematic uncertainty sources (fuel and moderator temperatures, methodological approximations in the CYCLE2008 calculation scheme, the impact of modelling the up-scattering phenomenon).

The experimental matrix is depicted in Figure 1. 


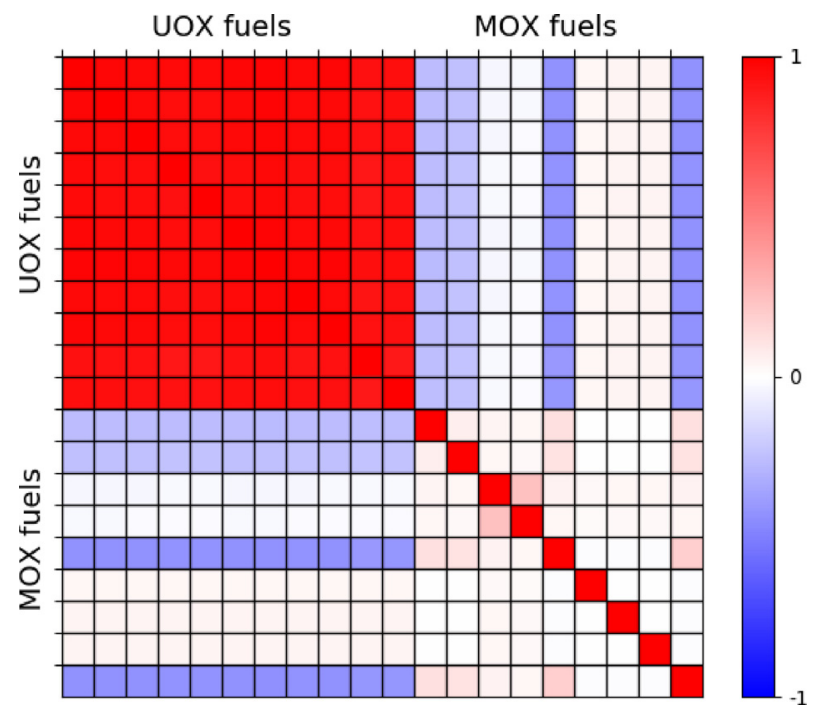

Fig. 1. Experimental correlation matrix obtained with the AGS code method, taking into account scheme-related uncertainties.

\subsection{Fitting method}

The fitting procedure implemented in the CONRAD code [15] relies upon the use of the generalized Bayes' theorem on conditional probability:

$$
P(\vec{x} \mid \vec{E}, U)=\frac{P(\vec{x}, U) \cdot P(\vec{E} \mid \vec{x}, U)}{\int P(\vec{x}, U) \cdot P(\vec{E} \mid \vec{x}, U) d \vec{x}},
$$

where:

$-\vec{x}$ represents the model parameters vector,

- $\vec{E}$ represents the experimental values vector,

- $U$ denotes the "prior" information,

- $P(\vec{x}, U)$ is the "prior" probability density,

- $P(\vec{E} \mid \vec{x}, U)$ is the "likelihood" probability density, corresponding to the Calculation-to-Experimental values,

- $P(\vec{x} \mid \vec{E}, U)$ represents the "posterior" probability density.

To solve this problem and determine the "posterior" probability density of the model parameters, assumptions on the "prior" distribution are necessary: according to the principle of maximum entropy, a multivariate joint normal distribution is chosen for the prior probability density.

If the Laplace approximation is made, the posterior density probability is assumed to be a normal distribution as well: the evaluation of the posterior parameters is achieved by finding the minimum of the following generalized least square function:

$$
\begin{aligned}
\chi^{2}= & \left(\vec{x}-\vec{x}_{m}\right)^{T} \cdot M_{x}^{-1} \cdot\left(\vec{x}-\vec{x}_{m}\right) \\
& +(\vec{C}-\vec{E})^{T} \cdot M_{E}^{-1} \cdot(\vec{C}-\vec{E}),
\end{aligned}
$$

where $\vec{x}_{m}$ designates the prior parameters and $M_{x}$ designates their associated covariance matrix. A GaussNewton iterative scheme is used [16] to solve equation (4) and derive the posterior model parameters and the associated covariance matrix. Nevertheless, only one iteration of this scheme can be used in this very specific study. Given that the overall give relevant results.

\subsection{Marginalization procedure}

A marginalization analysis is led in this study, to produce more realistic uncertainties associated to the posterior model parameters. This method [17] was implemented in CONRAD, to account for the uncertainties on nuisance parameters $\theta$. It consists in building a "full" $M$ covariance matrix of the simulation as follows:

$$
M=G^{T} \cdot \Sigma \cdot G,
$$

with:

$-\Sigma=\left(\begin{array}{cc}\Sigma_{x, x} & \Sigma_{x, \theta} \\ \Sigma_{\theta, x} & \Sigma_{\theta, \theta}\end{array}\right)=$ covariance matrix between fitted and nuisance parameters,

$-G=\left(\begin{array}{l}G_{x} \\ G_{\theta}\end{array}\right)=$ sensitivity vector of fitted and nuisance parameters.

One can deduce the $M_{x, M \text { arg }}$ covariance matrix for fitted parameters that reproduces the system when resolving:

$$
G_{x}^{T} \cdot M_{x, \text { Marg }} \cdot G_{x}=G^{T} \cdot \Sigma \cdot G .
$$

To avoid Peelle's pertinent puzzles [18], namely the occurrence of abnormal values of quantities that are fitted on experimental data with both statistical and systematic uncertainties, the burnup uncertainty (neodymium fission yields) was marginalized.

\section{Application of the method to ${ }^{137} \mathrm{Cs}$}

\section{$3.1{ }^{137} \mathrm{Cs}$ fitted parameters}

Figure 2 depicts the ${ }^{137} \mathrm{Cs}$ decay path. Each nuclide of this filiation is created through fissions on actinides like ${ }^{235} \mathrm{U}$, ${ }^{239} \mathrm{Pu},{ }^{241} \mathrm{Pu}$.

Given this decay path, one can list the model parameters used for the ${ }^{137} \mathrm{Cs}$ study. Either independent or cumulated fission yields could be considered here, provided that a prior correlation matrix between the independent fission yields is accounted. Recent studies have been conducted to compute these matrices [19].

In this paper, only the case of thermal cumulated fission yields will be presented. Considering that ${ }^{137} \mathrm{Cs}$ is mainly underestimated in UOX fuels at low and high combustion rate, it was decided to fit both ${ }^{235} \mathrm{U}$ and ${ }^{239} \mathrm{Pu}$ fission yields.

The prior uncertainty considered for these parameters are taken from COMAC-V2.0 $[19,20]$ and are $1.5 \%$ and $1.4 \%$ for ${ }^{235} \mathrm{U}$ and ${ }^{239} \mathrm{Pu}$ fission yields respectively. 


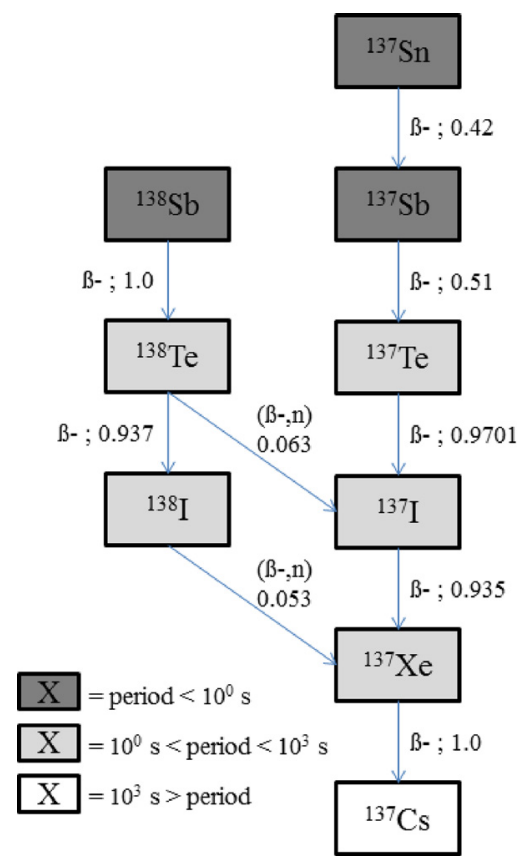

Fig. 2. Decay path of ${ }^{137} \mathrm{Cs}$ and associated branching ratios taken from JEFF-3.1.1.

A diagonal matrix was considered as prior correlation matrix between these parameters.

\subsection{Marginalized parameters}

Table 2 presents the model parameters used for this study and their associated uncertainties, in which $C_{X}^{Z}$ designates the $Z$ nuclide thermal cumulated fission yield in $X$ fission product, and $\tau(X)$ designates the $X$ period.

It can be noted that the impact of fission and capture cross sections of major actinides on ${ }^{137} \mathrm{Cs}$ buildup was taken into account. In this study, only 1-group cross sections were considered: the COMAC database was used to provide an uncertainty for 1-group cross sections, folding the covariance matrices as follows [21]:

$$
\operatorname{var}\left(\sigma_{1 g}\right)=\left(\frac{\tau_{i}}{\tau}\right)_{1 \leq i \leq n}^{T} . M_{\sigma} \cdot\left(\frac{\tau_{i}}{\tau}\right)_{1 \leq i \leq n}
$$

where:

$-\tau_{i}$ is the microscopic reaction rate of the $i$ th group,

$-\tau$ is the microscopic reaction rate,

$-\sigma$ is the reaction cross section of interest,

- $M_{\sigma}$ is the $n$-group covariance matrix of $\sigma$, with $n=26$.

Energy released by neutron reactions such as capture or fission were not accounted here as model parameters. Since they should be considered, it might induce a slight underestimation of the final uncertainty after the marginalization procedure. Further study should investigate this effect.

The marginalized parameters are assumed here to be independent.
Table 2. Model parameters used for the study, and associated uncertainties from COMAC-V2.0.

\begin{tabular}{llc}
\hline & \multicolumn{2}{l}{ Model parameters and uncertainties } \\
\hline Fitted & $C_{\mathrm{CS}-137}^{\mathrm{U}-235}$ & $1.5 \%$ \\
& $C_{\mathrm{Cs}-137}^{\mathrm{P}-239}$ & $1.4 \%$ \\
Marginalized & $\mathrm{U}-235(n, f)$ & $0.33 \%$ \\
& $\mathrm{U}-238(n, \gamma)$ & $0.85 \%$ \\
& $\mathrm{Pu}-239(n, \gamma)$ & $2.3 \%$ \\
& $\mathrm{Pu}-239(n, f)$ & $1.3 \%$ \\
& $\mathrm{Pu}-240(n, \gamma)$ & $1.9 \%$ \\
& $\mathrm{Pu}-241(n, \gamma)$ & $3.3 \%$ \\
& $\mathrm{Pu}-241(n, f)$ & $1.5 \%$ \\
& $C_{\mathrm{Cs}-137}^{\mathrm{Pu}-241}$ & $1.0 \%$ \\
& $\tau(\mathrm{Cs}-137)$ & $0.10 \%$ \\
& Burnup & $2.0 \%$ \\
\hline
\end{tabular}

\subsection{Sensitivity calculation}

A direct method to compute sensitivity coefficients was used with APOLLO2 and the CYCLE2008 calculation scheme: for each model parameter, a nominal calculation and a perturbed calculation are performed, with a $1 \%$ perturbation rate being applied to the parameter of interest for the latter. A routine was used to compute all sensitivity coefficients for all model parameters as follows:

$$
G_{x_{i, j}}=\left(\frac{C_{i} \cdot\left(1.01 \times x_{j}\right)-C_{i}\left(x_{j}\right)}{C_{i}\left(x_{j}\right) \times 0.01}\right) .
$$

The perturbation rate was applied after the self-shielding step of the first transport calculation. Self-shielded cross sections are chosen not to be re-calculated at specific burnup steps, contrary to the CYCLE2008 recommendations, in order not to overwrite the perturbation rate. This modification of the calculation scheme on fuel inventory has a very small impact, which becomes negligible on sensitivity coefficients, provided that self-shielded cross sections are not re-calculated in the nominal calculation either.

In this study, the fact that covariance matrices are associated to infinite diluted cross-sections instead of selfshielded cross-sections is not assessed. Since no crosssection is fitted in this study, this will only affect the uncertainty associated to fitted fission yields through the marginalization process. The impact of this effect will be investigated in further study, and might increase the final uncertainty after marginalization.

Table 3 depicts the sensitivity coefficients for the chosen model parameters for three types of irradiated fuel to give general tendencies on the sensitivity coefficients. 
Table 3. Model parameters used for the study and their sensitivity coefficient for a $3.1 \%$-enriched UOX fuel at 20 and $60 \mathrm{GWd} / \mathrm{t}$, and a $6.7 \% \mathrm{Pu}$ amount $\mathrm{MOX}$ fuel at $40 \mathrm{GWd} / \mathrm{t}$.

\begin{tabular}{lccc}
\hline \multicolumn{1}{c}{ Model parameters } & \multicolumn{3}{c}{ Sensitivity coefficients } \\
\cline { 2 - 4 } & $\begin{array}{l}3.1 \% \text {-enriched UOX fuel } \\
20 \mathrm{GWd} / \mathrm{t}\end{array}$ & $\begin{array}{l}3.1 \% \text {-enriched UOX fuel } \\
60 \mathrm{GWd} / \mathrm{t}\end{array}$ & $\begin{array}{l}6.7 \% \mathrm{Pu} \text { amount MOX fuel } \\
40 \mathrm{GWd} / \mathrm{t}\end{array}$ \\
\hline Fitted & & \\
$C_{\mathrm{Cs}-235}^{\mathrm{U}-137}$ & 0.62 & 0.35 & 0.01 \\
$C_{\mathrm{Cs}-137}^{\mathrm{Pu}-239}$ & 0.27 & 0.44 & 0.63 \\
$\mathrm{Marginalized}$ & & & \\
$\mathrm{U}-235(n, f)$ & 0.01 & 0.01 & 0.00 \\
$\mathrm{U}-238(n, \gamma)$ & -0.01 & -0.01 & -0.01 \\
$\mathrm{Pu}-239(n, \gamma)$ & -0.01 & -0.02 & -0.02 \\
$\mathrm{Pu}-239(n, f)$ & -0.01 & 0.02 & 0.03 \\
$\mathrm{Pu}-240(n, \gamma)$ & 0.00 & 0.00 & -0.01 \\
$\mathrm{Pu}-241(n, \gamma)$ & 0.00 & 0.00 & 0.00 \\
$\mathrm{Pu}-241(n, f)$ & 0.00 & 0.00 & 0.01 \\
$C_{\mathrm{Cs}-137}^{\mathrm{Pu}-241}$ & 0.02 & 0.10 & 0.23 \\
$\tau(\mathrm{Cs}-137)$ & -0.14 & -0.17 & -0.10 \\
Burnup & 1.0 & 1.5 & 1.0 \\
\hline
\end{tabular}

Table 4. Results of the DARWIN2.3 integral data assimilation on ${ }^{235} \mathrm{U}$ and ${ }^{239} \mathrm{Pu}$ fission yields.

Model parameters and uncertainties

\begin{tabular}{|c|c|c|}
\hline $\begin{array}{l}\text { Parameters } \\
\text { Prior ( } 1 \sigma \text {-uncertainty) }\end{array}$ & $C_{\mathrm{Cs}-137}^{\mathrm{U}-235}$ & $\begin{array}{l}C_{\mathrm{Cs}-137}^{\mathrm{Pu}-239} \\
6.59 \mathrm{E}-02 \pm 1.4 \%\end{array}$ \\
\hline Prior (1 $\sigma$-uncertainty) & $6.22 \mathrm{E}-02 \pm 1.5 \%$ & $\begin{array}{l}6.59 \mathrm{E}-02 \pm 1.4 \% \\
6.62 \mathrm{E}-02+0.2 \%\end{array}$ \\
\hline 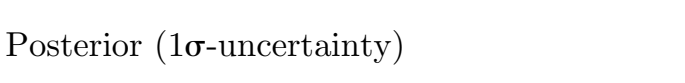 & $\begin{array}{l}6.46 \mathrm{E}-02 \pm 1.1 \% \\
(+3.8 \pm 1.1 \%)\end{array}$ & $\begin{array}{l}6.62 \mathrm{E}-02 \pm 0.2 \% \\
(+0.5 \pm 0.2 \%)\end{array}$ \\
\hline Posterior + Marginalization ( $1 \sigma$-uncertainty) & $\begin{array}{l}6.46 \mathrm{E}-02 \pm 2.1 \% \\
(+3.8 \pm 2.1 \%)\end{array}$ & $\begin{array}{l}6.62 \mathrm{E}-02 \pm 3.3 \% \\
(+0.5 \pm 3.3 \%)\end{array}$ \\
\hline
\end{tabular}

One can see that the thermal cumulative fission yield of ${ }^{239} \mathrm{Pu}$ in ${ }^{137} \mathrm{Cs}$ has an important sensitivity for UOX fuels even at low burnup. This also justifies fitting it together with the ${ }^{235} \mathrm{U}$ thermal cumulative fission yield.

\section{Results and discussion}

Table 4 displays the results obtained when fitting both ${ }^{235} \mathrm{U}$ and ${ }^{239} \mathrm{Pu}$ cumulated thermal fission yields in ${ }^{137} \mathrm{Cs}$.

One can observe an increase of the ${ }^{235} \mathrm{U}$ cumulated thermal fission yield in ${ }^{137} \mathrm{Cs}$, consistent with the underestimation of its concentration in UOX fuels. The marginalization procedure computes more realistic uncertainties for both fission yields, as one can see on Table 4 .

The study does not suggest a change in ${ }^{239} \mathrm{Pu}$ cumulated thermal fission yields in ${ }^{137} \mathrm{Cs}$, given its very small modification, consistent with the good calculation of ${ }^{137} \mathrm{Cs}$ concentration in MOX fuels.

Figure 3 depicts the $(\mathrm{C} / \mathrm{E})-1$ values calculated by CONRAD for UOX fuels before adjustment, after the adjustment step, and after the full process of adjustment and marginalization. One can see a satisfactory compensation of the underestimated prior values. The one-iteration limitation on the cost function minimization process does not seem to be an issue here, since consistent results are obtained.

The residual slight underestimation of $(\mathrm{C} / \mathrm{E})-1$ is due to the relative small prior uncertainty of the ${ }^{235} \mathrm{U}$ cumulated thermal fission yield in ${ }^{137} \mathrm{Cs}$, therefore constraining the posterior value.

It is worth comparing the ${ }^{235} \mathrm{U}$ cumulated thermal fission yields in ${ }^{137} \mathrm{Cs}$ value with different libraries. One can observe on Figure 4 that this work is consistent within the uncertainties with JEFF-3.1.1 at $1 \sigma$ and other evaluations at $2 \sigma$.

However it is important to point out that JEFF-3.3 suggests the opposite of the present work, meaning that the latest JEFF evaluation will amplify the current underestimation of the ${ }^{137} \mathrm{Cs}$ concentration calculation with the DARWIN2.3 package.

To complete this study, it will be interesting to confront the result obtained with microscopic measurements. 

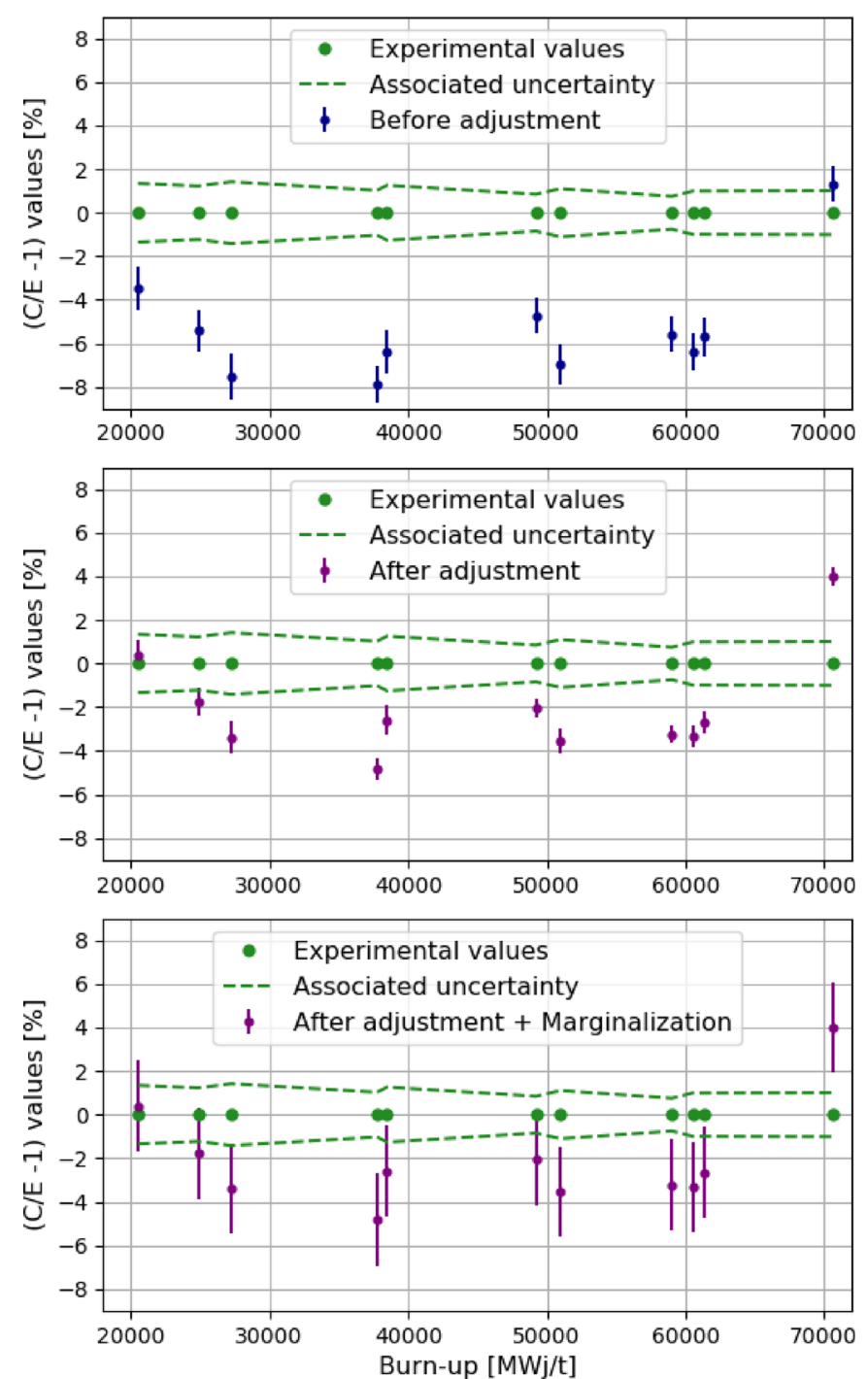

Fig. 3. (C/E)-1 values of the ${ }^{137} \mathrm{Cs}$ concentration calculation before fit (top), after fit (middle), and after fit + marginalization (bottom).

\section{Conclusion}

The assimilation of integral data from the DARWIN2.3 package experimental validation, using JEFF-3.1.1 library, was investigated here.

The AGS code method was used to provide an experimental correlation matrix between the PIEs, allowing to take into account both the statistic and systematic sources of uncertainty. The order of magnitude of numerical biases on fuel inventory calculation were also quantified and considered as $1 \sigma$ uncertainty on fuel inventory calculation.

The generalized least-square equation derived from Bayes' theorem was used to fit both ${ }^{235} \mathrm{U}$ and ${ }^{239} \mathrm{Pu}$ cumulated thermal fission yields in ${ }^{137} \mathrm{Cs}$.

Uncertainties on nuisance parameters involved in ${ }^{137} \mathrm{Cs}$ buildup, e.g. energy-integrated capture or fission cross section of actinides, are accounted through an analytic

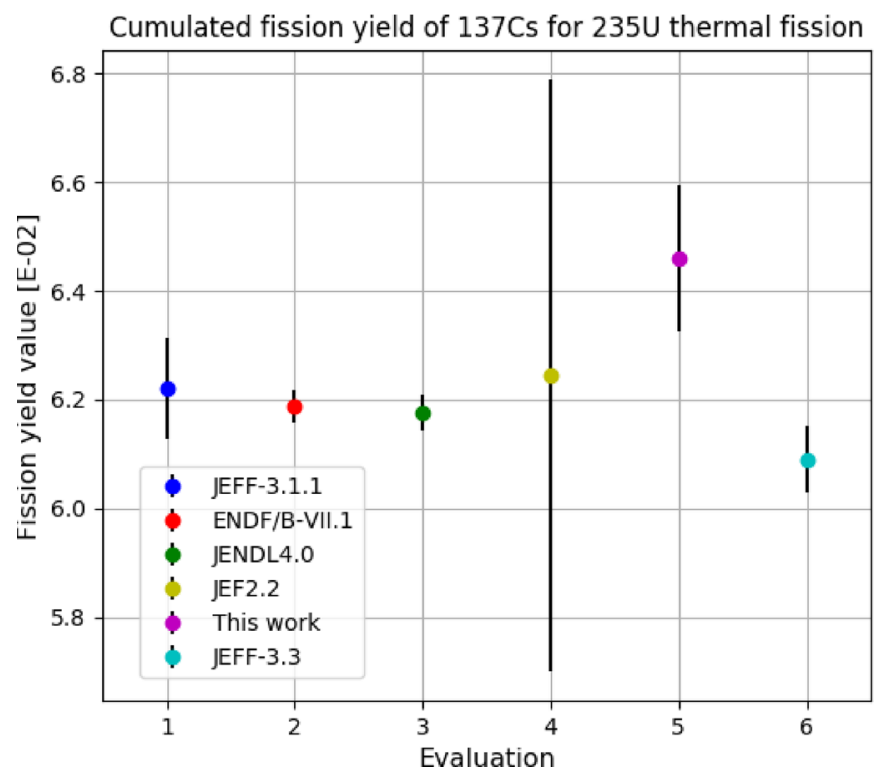

Fig. 4. ${ }^{235} \mathrm{U}$ cumulated thermal fission yields in ${ }^{137} \mathrm{Cs}$ from various nuclear data evaluation $(1 \sigma)$.

marginalization technique implemented in the CONRAD code, leading to more realistic uncertainty on the posterior values of the fitted parameters.

Results suggest an increase of the ${ }^{235} \mathrm{U}$ cumulated thermal fission yields in ${ }^{137} \mathrm{Cs}$ by $+(3.8 \pm 2.1) \%$, from $6.22 \mathrm{E}-02$ in JEFF-3.1.1 to $6.46 \mathrm{E}-02 \pm 2.1 \%$. This adjusted value of ${ }^{235} \mathrm{U}$ cumulated thermal fission yields in ${ }^{137} \mathrm{Cs}$ leads to reduced $(\mathrm{C} / \mathrm{E})-1$ values.

Although the posterior fission yield value is consistent with other international nuclear data libraries at $2 \sigma$, one can point out that the latest JEFF-3.3 suggests the opposite of the present work.

Even though the method is validated here, one can emphasize the importance of reliable and dedicated integral data experiments to fit nuclear data.

Further studies are expected to be led with the same method to assimilate more integral data from the DARWIN2.3 package experimental validation and investigate other nuclides important for the fuel cycle.

We would like to thank all the colleagues from CEA who helped us during this work, especially, David Bernard, Coralie Carmouze, Pascal Archier, Stéphane Mengelle and Claude Mounier.

\section{Author contribution statement}

Axel Rizzo carried out all the calculations for the study reported in this paper. Claire Vaglio-Gaudard and Gilles Noguère provided help and pieces of advice for the analysis of the results, as well as support for handling the CONRAD code. Many fruitful discussions were conducted with JulieFiona Martin and Romain Eschbach, and provided valuable knowledge for the understanding of the fuel cycle. Vanessa Vallet also provided pieces of advice for the use of the CYRUS code. 


\section{References}

1. J.F. Martin et al., EPJ Web Conf. 146, 09010 (2017)

2. L. San-Felice et al., Nucl. Tech. 184, 217 (2013)

3. A. Santamarina et al., The JEFF-3.1.1 Nuclear Data library, JEFF report, 22, OECD-NEA data bank, 2009

4. A. Santamarina et al., Validation of the new code package APOLLO2.8 for accurate PWR neutronics calculations, in Proc. Int. Conf. Advances in Nuclear Fuel Management IV, Hilton Head Island, South Carolina, USA, 2009

5. J.M. Ruggieri et al., Eranos2.1: the International Code System for GEN-IV fast reactor analysis, in Proc. Int. Conf. ICAPP, Reno, Nevada, USA, 2006

6. A. Tsilanizara et al., DARWIN: an evolution code system for a large range of applications, in Proc. Int. Conf. ICRS-9, Tsukuba, Ibaraki, Japan, 1999

7. J.F. Lebrat et al., Analysis of the TRAPU and DOUBLON irradiations in PHENIX for the experimental validation of the DARWIN package for fast reactors, in Proc. Int. Conf. GLOBAL, Paris, France, 2015

8. A. Rizzo et al., EPJ Web Conf. 146, 09030 (2017)

9. C. de Saint Jean et al., Status of CONRAD, a nuclear reaction analysis tool, in Proc. Int. Conf. Nuclear Data for Science and Technology, Nice, France, 2007

10. P. Archier et al., CONRAD evaluation code: development status and perspectives, in Proc. Int. Conf. Nuclear Data for Science and Technology, New-York, USA, 2013
11. C. Bastian et al., AGS, a computer code for uncertainty propagation in time-of-flight cross section data, in Proc. Int. Conf. PHYSOR, Vancouver, Canada, 2006

12. E. Brun et al., Ann. Nucl. Energy 82, 151 (2015)

13. S. Lahaye et al., First verification and validation steps of MENDEL release 1.0 cycle code system, in Proc. Int. Conf. PHYSOR, Kyoto, Japan, 2014

14. M. Ouisloumen et al., Nucl. Sci. Eng. 107, 189 (1991)

15. E. Privas et al., EPJ Web Conf. 106, 04015 (2016)

16. B. Habert et al., Nucl. Sci. Eng. 166, 276 (2010)

17. G. Noguere et al., Nucl. Sci. Eng. 172, 164 (2012)

18. D.L. Smith, Probability, statistics, and data uncertainties in nuclear science and technology (American Nuclear Society, La Grange Park, Illinois, 1991)

19. N. Terranova, Covariance Evaluation for Nuclear Data of Interest to the Reactivity Loss Estimation of the Jules Horowitz Reactor, Ph.D. thesis report, Bologne University, Italy, 2016

20. P. Archier et al., COMAC - Nuclear data covariance matrices library for reactor applications, in Proc. Int. Conf. PHYSOR, Kyoto, Japan, 2014

21. G. Krivtchik, Analysis of uncertainty propagation in nuclear fuel cycle scenarios, Ph.D. thesis report, Grenoble University, France, 2014

Cite this article as: Axel Rizzo, Claire Vaglio-Gaudard, Gilles Noguere, Julie-Fiona Martin, Vanessa Vallet, Romain Eschbach, Nuclear data adjustment based on the interpretation of post-irradiation experiments with the DARWIN2.3 package, EPJ Nuclear Sci. Technol. 4, 47 (2018) 